\title{
Plasmon resonance spectroscopy: probing molecular interactions at surfaces and interfaces
}

\author{
Zdzislaw Salamon and Gordon Tollin* \\ Department of Biochemistry and Molecular Biophysics, University of Arizona, Tucson, AZ 85721, USA
}

\begin{abstract}
Surface plasmon resonance (SPR) spectroscopy can be applied to a wide variety of interfacial systems. It involves resonant excitation by polarized light of electronic oscillations (plasmons) in a thin metal film. These generate a surfacelocalized evanescent electromagnetic field that can be used to probe the optical properties perpendicular to the film plane of materials immobilized at the surface. Spectra depend on three parameters: refractive index $(n)$, absorption coefficient $(k)$ and thickness $(t)$. Maxwell's equations provide an analytical relationship between these properties and SPR spectra, allowing their evaluation. An extension of this methodology, called coupled plasmon-waveguide resonance (CPWR or PWR), is able to characterize film properties both perpendicular and parallel to the surface plane. In a PWR device, the metal film is covered with a dielectric coating that acts as an optical amplifier, provides protection for the metal layer, and possesses a surface that allows various molecular immobilization strategies. The exceptionally narrow line widths of PWR spectra yield enhanced sensitivity and resolution. The application of this technology to several biomembrane systems will be described, demonstrating its ability to observe both binding and structural events occurring during membrane protein function.
\end{abstract}

\section{Introduction}

Plasmon resonance spectroscopy is an optical technique for probing events occurring at surfaces and interfaces. It is based upon the excitation of plasmons (charge density oscillations) by light in thin metal films. This generates a surface-localized (evanescent) electromagnetic wave that propagates along the interface between the film and an adjacent dielectric medium (air, water, etc.). The electric field lies normal to this interface and decays exponentially with distance on both sides of the interface. Solid-state physicists have used this phenomenon to characterize the structural properties of thin metallic films and dielectric coatings, and physical chemists have used it to study metal-electrolyte surfaces and LangmuirBlodgett films. In recent years, plasmon spectroscopy has become widely used in the biosensor, pharmaceutical, analytical chemistry, and membrane biochemistry/biophysics communities [1-7].

It is well recognized that monolayer assemblies of molecules at surfaces and interfaces can exist as organized two-dimensional systems displaying long-range order. This important structural characteristic is one of the fundamental attributes that control physicochemical processes occurring within such systems. Biological membranes, which are the focus of this article, are an example of such ordered two-dimensional arrays. These are self-assembling proteolipid aggregates consisting of a lipid bilayer associated with a variety of integral (transmembrane) and peripheral (surface-bound) proteins. An important characteristic of biomembranes is their fluid nature, which, although dynamic, retains structural organization on microscopic or spectroscopic length scales. Furthermore, such two-dimensional order

\footnotetext{
*Corresponding author. Tel. +1 520621 3447; Fax: +1 520621 9288; E-mail: gtollin@u.arizona.edu.
} 
can undergo significant changes as a result of interactions between lipid and protein molecules occurring during membrane functional activity. Thus, spectroscopic techniques employed to study such complex anisotropic structures must be capable of assessing these characteristics. As will be demonstrated below, a recently developed variant of plasmon resonance spectroscopy, called coupled plasmon-waveguide resonance (CPWR or PWR) [8-10], combined with techniques for forming solid-supported proteolipid membranes, possesses several important advantages that make it especially well suited for kinetic, thermodynamic, and structural studies of biomembranes.

\section{Principles of plasmon resonance spectroscopy}

The physics of surface plasmons propagating along a metal/dielectric interface has been studied extensively, and their fundamental properties can be described by the plasma formulation of Maxwell's theory of electromagnetism. Plasmon excitation occurs only when energy and momentum conditions between incident photons and surface plasmons are matched, according to the following equation [9]:

$$
\kappa_{\mathrm{SP}}=\kappa_{\mathrm{ph}}=(\omega / c) \varepsilon_{0}^{1 / 2} \sin \alpha_{0},
$$

where

$$
\kappa_{\mathrm{SP}}=(\omega / c)\left(\varepsilon_{1} \varepsilon_{2} / \varepsilon_{1}+\varepsilon_{2}\right)^{1 / 2} .
$$

$\kappa_{\mathrm{SP}}$ is the longitudinal component of the surface plasmon wavevector, $\kappa_{\mathrm{ph}}$ is the component of the exciting light wavevector parallel to the active medium surface, $\omega$ is the frequency of the surface plasmon excitation wavelength, $c$ is the velocity of light in vacuo, $\varepsilon_{0}, \varepsilon_{1}$ and $\varepsilon_{2}$ are the complex dielectric constants for the incident, surface active and dielectric (or emerging) media, respectively, and $\alpha_{0}$ is the incident coupling angle. In order to satisfy this relationship in a conventional resonator assembly [8-10], plasmon excitation must occur via an evanescent wave generated by $p$-polarized incident light under total internal reflection conditions. Experimentally, this can be accomplished by depositing a metallic film on the external surface of a right angle prism that is illuminated at the critical angle. It is important to note that in $p$-polarization, the electric vector is parallel to the incident plane, which for large incident angles becomes perpendicular to the film plane; $s$-polarized light has the electric vector perpendicular to the incident plane, i.e., parallel to the film plane.

The resonance condition in Eq. (1a) can be fulfilled by either changing the incident angle, $\alpha$, at a constant value of photon energy, $h c / \lambda$, or by varying $\lambda$ at a constant value of the incident angle, $\alpha=\alpha_{0}$. The resulting variation in reflected light intensity with either incident angle or exciting light wavelength constitutes a surface plasmon resonance (SPR) spectrum [10]. Furthermore, any alteration in the optical properties of the metal/emerging medium interface will affect the $\kappa_{\mathrm{SP}}$ value and therefore change the resonance characteristics. These optical properties can be fully described by the complex dielectric constant, $\varepsilon$, in Eq. (1b), which contains the refractive index, $n$, and the extinction coefficient, $k$, i.e., $\varepsilon=n-$ $\mathrm{i} k$. Thus, deposition of a thin dielectric film on the metal surface introduces changes in the optical parameters of the metal/emerging medium interface, thereby causing a shift of the surface plasmon wavevector to a larger value. According to Eq. (1a), such a shift moves the resonance to a larger incident angle. An example of this is given in Fig. 1. As is evident, the spectral line shape (width and amplitude) is also dramatically altered. 


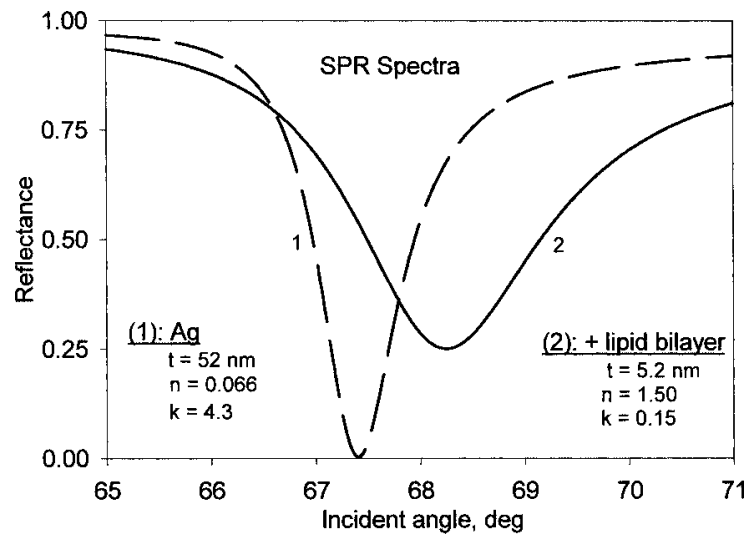

Fig. 1. Theoretical SPR spectra calculated for a silver film in contact with an aqueous medium in the absence (curve 1) and in the presence of a deposited lipid membrane (curve 2). Optical parameters of silver and lipid layers are as shown $(\lambda=632.8 \mathrm{~nm})$.

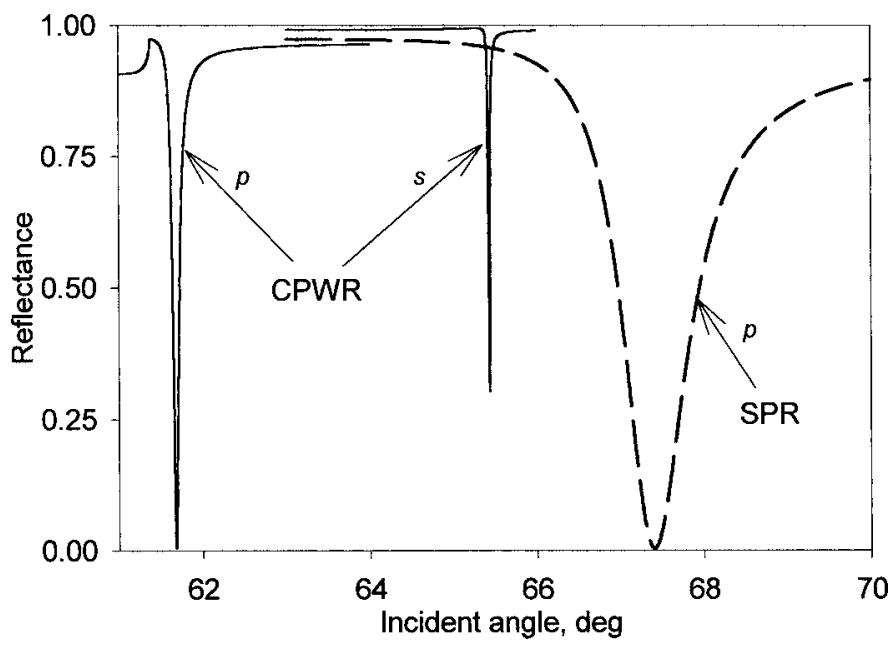

Fig. 2. Comparison between theoretical SPR and CPWR spectra calculated for a silver film in contact with an aqueous medium $(\lambda=632.8 \mathrm{~nm})$. Exciting light polarizations are as shown.

Coupled plasmon-waveguide resonance [8] involves more complex assemblies in which surface plasmon resonance in a thin metal film is coupled with guided waves in an adjacent dielectric layer, resulting in excitation of two different resonances depending on incident light polarization. A PWR resonator contains a metallic layer that is deposited on a prism (the same as in a conventional SPR assembly), but is overcoated with either a single dielectric layer or a system of dielectric layers. The added layers are characterized by appropriate optical parameters so that the assembly is able to generate surface resonances upon excitation by both $p$ - and $s$-polarized light components. A comparison of the spectra obtained with SPR and PWR resonators is shown in Fig. 2.

The addition of such a dielectric layer (or layers) to a conventional SPR assembly plays several important roles [8-10]. First, it functions as an optical amplifier that significantly increases electromagnetic field intensities at the dielectric surface in comparison to conventional SPR. This results in an increased sensitivity and spectral resolution (the latter due to decreased resonance linewidths). Second, it enhances spectroscopic capabilities (due to excitation of resonances with both $p$ - and $s$-polarized light 

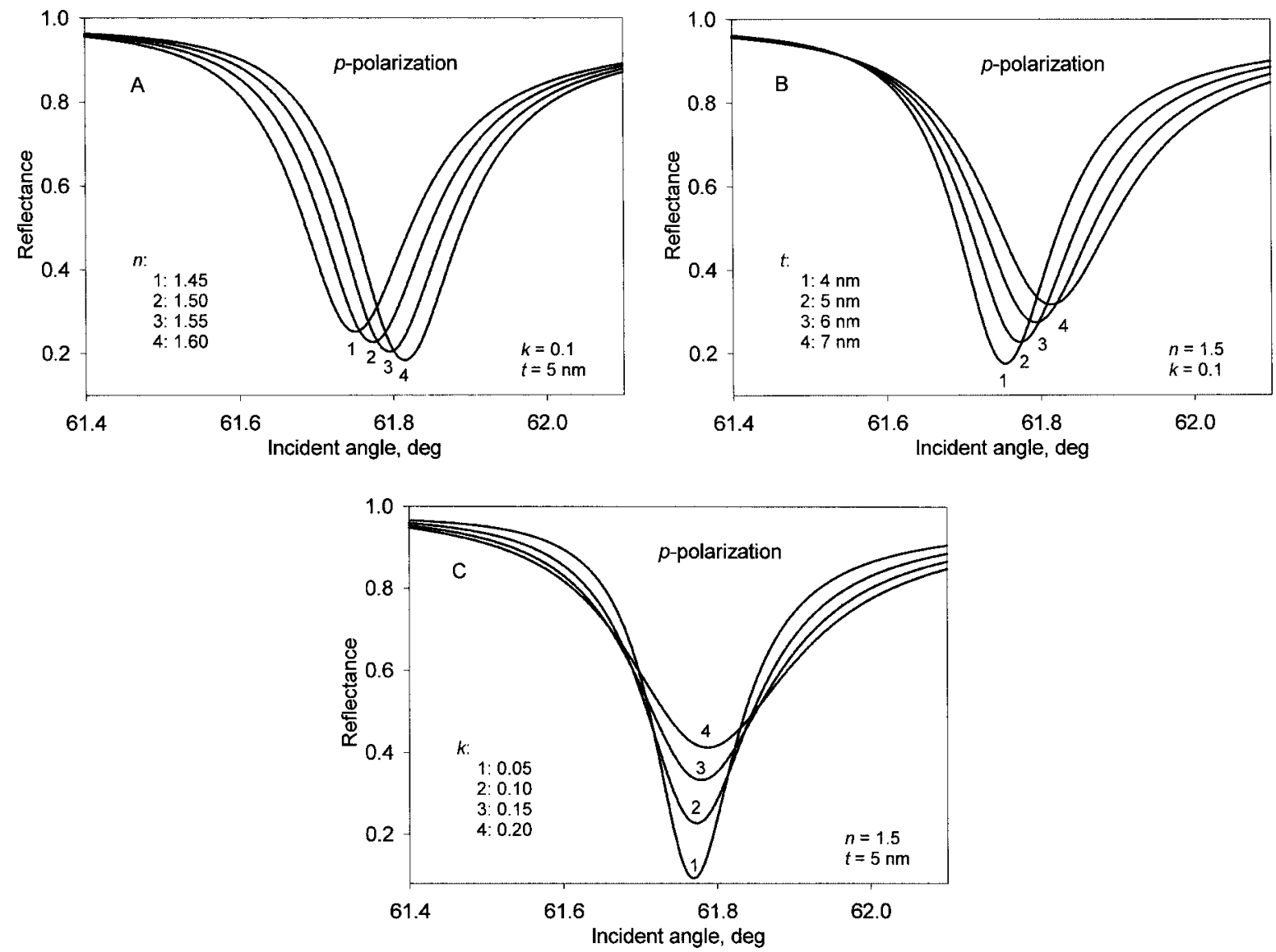

Fig. 3. Effect of changes in optical parameters on theoretical CPWR spectra obtained for a deposited lipid membrane with $p$-polarized exciting light. (A) changes in refractive index, $n$; (B) changes in thickness, $t$; (C) changes in optical absorption coefficient, $k$.

components), which results in the ability to directly measure anisotropies in refractive index and optical absorption coefficient in thin films adsorbed onto the surface of the overcoating. Third, the dielectric overcoating serves as a mechanical and chemical shield for the thin metal layer.

The position, the width, and the depth of an SPR or PWR spectrum are determined by the optical parameters $n$ and $k$ and by the film thickness $(t)$. The influence of these parameters on the spectrum can be analyzed by applying Maxwell's equations to thin film assemblies [9-11]. It is important to note that the number of measured spectral parameters equals the number of unknown optical parameters. As demonstrated in Figs 3 and 4, the three parameters are well separated in their effects on the plasmon resonance spectra. This allows a unique determination of their values by fitting a theoretical resonance curve to the experimental one. Note that for an anisotropic thin film, such as a proteolipid membrane, $t$ represents an average molecular length perpendicular to the plane of the film, and will be independent of light polarization. In contrast, the values of $n$ and $k$ will be very much dependent on the polarization of the excitation light. In PWR spectroscopy, this results in two values of the refractive index $\left(n_{\mathrm{p}}\right.$ and $\left.n_{\mathrm{s}}\right)$, two values of the extinction coefficient $\left(k_{\mathrm{p}}\right.$ and $k_{\mathrm{s}}$ ) and a single value of the film thickness. Furthermore, for uniaxial anisotropic structures in which the optical axis is parallel to the $p$-polarization direction, the $n_{\mathrm{p}}$ value will always be larger than $n_{\mathrm{s}}$ [12]. This is a consequence of the fact that the measured refractive 

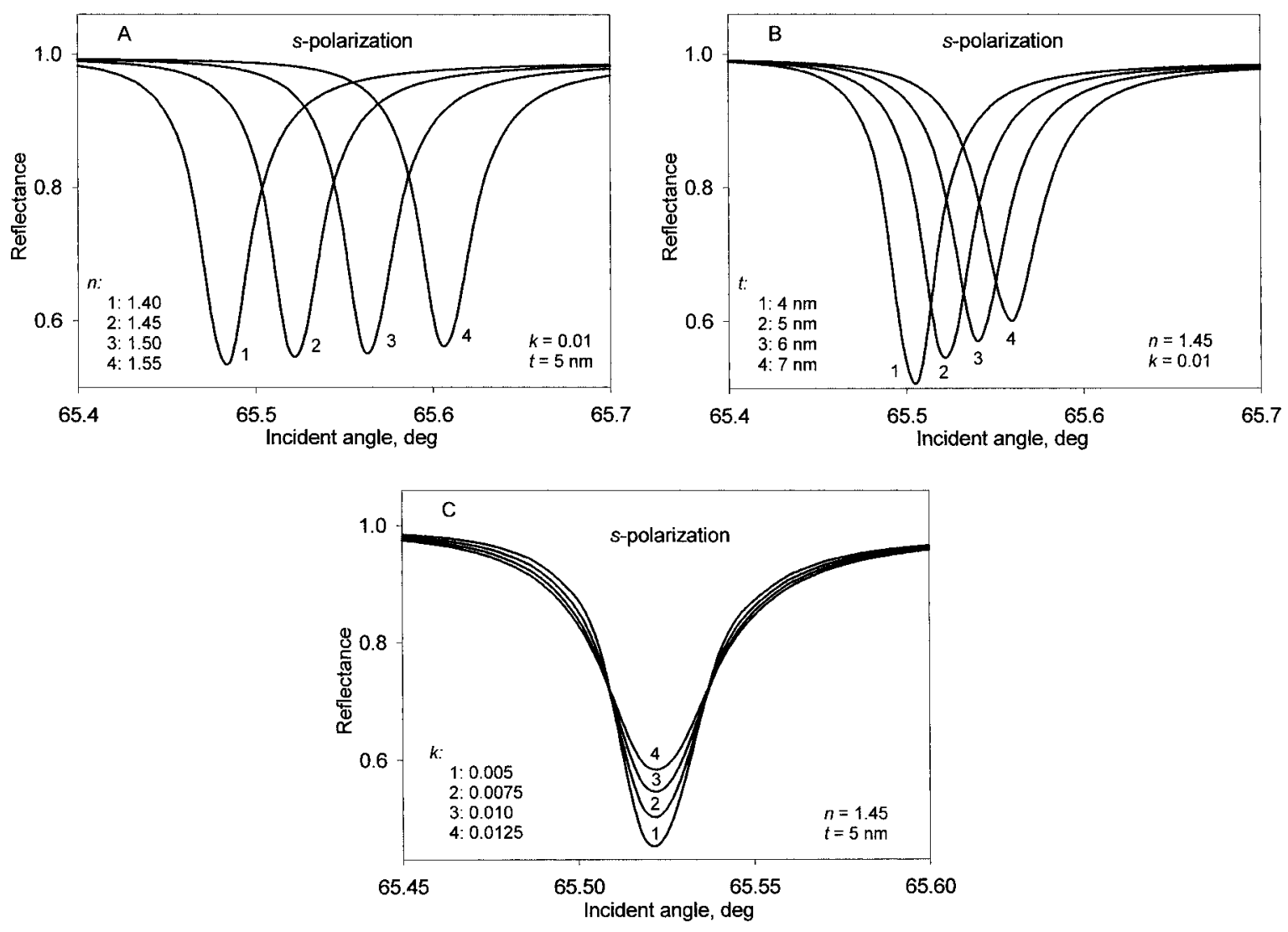

Fig. 4. Effect of changes in optical parameters on theoretical CPWR spectra obtained for a deposited lipid membrane with $s$-polarized exciting light. (A) changes in refractive index, $n$; (B) changes in thickness, $t$; (C) changes in optical absorption coefficient, $k$.

index of a material is determined by the polarizability of the individual molecules. The latter property describes the ability of a molecule to interact with an external electromagnetic field, and in general is anisotropic with respect to the molecular frame. In the simplified case in which the molecular shape is rod-like (e.g., as in the phospholipid molecules forming a biomembrane), one can assign two different values to the polarizability: the larger one, longitudinal and the smaller one, transverse. If in addition to the anisotropy in molecular shape and polarizability, the system that contains these molecules is ordered such that the long axes of the molecules are parallel, this results in long-range order usually described by the order parameter $S$ [12]. In this situation the values of the polarizability, averaged over the whole system and measured either parallel or perpendicular to the direction of the long axis of the molecules, will be different (i.e., the parallel value will be larger than the perpendicular one). These conditions create an optically anisotropic system, with the optical axis perpendicular to the plane of the proteolipid membrane, and the values of the refractive index measured with two polarizations of light (i.e., parallel, $n_{\mathrm{p}}$, and perpendicular, $n_{\mathrm{s}}$ to the optical axis) will describe this optical anisotropy $\left(A_{\mathrm{n}}\right)$ [12].

The parameters obtained from fitting PWR spectra can be used to calculate the anisotropy of $n$ and $k$, thereby describing the degree of both molecular order (by the anisotropy in $n$ ) and orientation of chromophore groups attached to the molecules comprising the thin film (by the anisotropy in $k$ ), using the 
following relationships [12]:

$$
\begin{aligned}
& n_{\mathrm{av}}^{2}=\left(n_{\mathrm{p}}^{2}+2 n_{\mathrm{s}}^{2}\right) / 3=\text { average value of the refractive index, } \\
& k_{\mathrm{av}}=\left(k_{\mathrm{p}}+2 k_{\mathrm{s}}\right) / 3=\text { average value of the extinction coefficient, } \\
& A_{\mathrm{n}}=\left(n_{\mathrm{p}}^{2}-n_{\mathrm{s}}^{2}\right) /\left(n_{\mathrm{av}}^{2}+2\right)=\text { refractive index anisotropy, } \\
& A_{\mathrm{k}}=\left(k_{\mathrm{p}}-k_{\mathrm{s}}\right) / k_{\mathrm{av}}=\text { extinction coefficient anisotropy. }
\end{aligned}
$$

There are two situations in which the extinction coefficient anisotropy can provide useful information: (1) If the molecules are macroscopically aligned (as is the case in biomembranes) then the orientation of transition dipoles can be determined with respect to the molecular coordinates. (2) When the orientation of a transition dipole is known with respect to the molecular coordinates, then the anisotropy provides information on the orientation of the molecule with respect to the symmetry axis of the sample.

Such information, taken together with the film thickness, provides insights into the microscopic structure of the film. Furthermore, the optical parameters can also be employed to calculate the mass of a deposited thin layer, using the Lorentz-Lorenz relation for a film containing a mixture of substances, or of a chromophore using the Lambert-Beer relationship [9,11,13]:

$$
\begin{aligned}
& d=M L=(M / A)\left[\left(n_{\mathrm{av}}^{2}-1\right) /\left(n_{\mathrm{av}}^{2}+2\right)\right]=\text { mass density, } \\
& C=(4 \pi / \lambda)\left(k_{\mathrm{av}} / \beta\right)=\text { surface concentration of chromophore. }
\end{aligned}
$$

In these equations $M=$ molecular weight, $L=$ number of moles per unit volume, $A=$ molar refractivity, $C=$ molar concentration and $\beta=$ molar absorptivity.

Plasmon spectroscopy utilizes a simple optical system involving the measurement of reflected light intensity. This is shown in Fig. 5. The geometrical arrangement permits a complete isolation of the exciting light from the system under investigation. Thus, photochemically active mixtures or optically dense media can be studied. Inasmuch as the methodology involves the use of evanescent waves that are generated under resonance conditions, an exceptionally high sensitivity is obtained. A further advantage is that spectral information can be obtained rapidly and changes can be monitored in real time. A potential

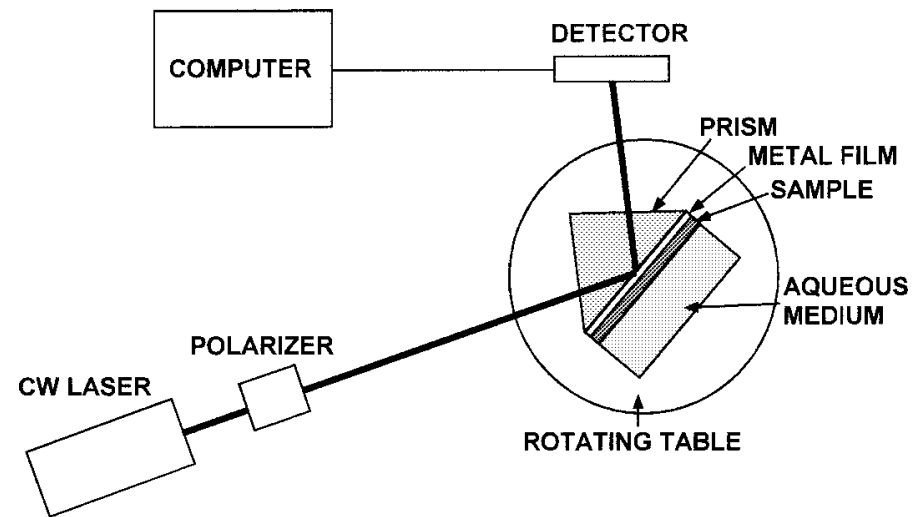

Fig. 5. Schematic view of a plasmon resonance spectrometer. 
drawback to the investigation of biological systems is that plasmon spectroscopy requires the immobilization of molecules at a solid surface. It is thus necessary to ensure that such immobilization does not alter the biochemical characteristics of the materials.

\section{Application of PWR spectroscopy to biomembrane systems}

\subsection{Formation of lipid membranes}

Self-assembled solid-supported lipid membranes can be generated on the resonator surface using a variation of the method used for formation of freely suspended lipid bilayers [14]. This involves spreading a small amount of lipid solution across an orifice in a Teflon sheet that separates the thin dielectric film $\left(\mathrm{SiO}_{2}\right)$ from the aqueous phase in the PWR cell [9]. The hydrophilic surface of hydrated $\mathrm{SiO}_{2}$ attracts the polar groups of the lipid molecules, thus inducing an initial orientation of the lipid molecules, with the hydrocarbon chains pointing toward the droplet of excess lipid solution. The next steps of bilayer formation, induced by adding aqueous buffer into the sample compartment of the PWR cell, involve a thinning process and formation of a plateau-Gibbs border of lipid solution that anchors the membrane to the Teflon spacer. This border allows lipid molecules to either enter or leave the bilayer in response to changes such as insertion of proteins or macromolecular conformational transitions.

\subsection{Insertion of proteins into a preformed bilayer}

Integral membrane protein molecules can be incorporated into a preformed lipid membrane deposited on the hydrophilic surface of the silica film. This is done by adding small aliquots of a concentrated solution of the protein solubilized in a detergent-containing buffer (usually $30 \mathrm{mM}$ octyl glucoside) to the aqueous volume of the PWR cell that is in contact with the bilayer. This dilutes the detergent to a final concentration below its critical micelle concentration ( $25 \mathrm{mM}$ in the case of octyl glucoside) [15-18], resulting in spontaneous transfer of the protein from the detergent micelle to the lipid bilayer.

\subsection{Calibration of PWR resonator and analysis of spectra}

The plasmon-generating device can be calibrated by measuring the PWR spectra obtained from a bare silica surface in contact with an aqueous medium with both $p$ - and $s$-polarized light, and then fitting these with theoretical curves. The goal of such a calibration is to obtain the optical parameters of the silver and silica layers $[10,11,13]$. This provides an input data set used in analyzing the resonance spectra obtained with proteolipid membranes deposited on the silica surface. Thus, the resonance spectra obtained after a lipid bilayer membrane is created on the hydrophilic surface of silica can be fit using these data, yielding the optical parameters for the lipid bilayer. These values allow the calculation of the refractive index anisotropy and the surface mass density (i.e., molecular packing density) of the bilayer. After incorporation of protein molecules into the lipid membrane, the resulting PWR spectra can be used to characterize the structural consequences of protein insertion into the bilayer by fitting procedures. Finally, addition of soluble molecules that bind to the inserted protein into the aqueous sample compartment of the PWR cell again results in changes of the PWR spectra, which reflect structural alterations in the proteolipid membrane caused by the protein-ligand interaction. These can again be characterized using spectral fitting. 
All PWR experiments described below were carried out using red light from a CW He-Ne laser. Inasmuch as none of the molecules investigated here has specific absorption bands at this wavelength, the $k$ parameter does not contribute significantly to the PWR spectra in these applications.

\section{Specific examples of applications of plasmon resonance to membrane proteins}

We will describe our plasmon spectroscopy studies of two integral membrane proteins, one of which is involved in photosynthetic energy transduction, cytochrome $b_{6} f\left(c y t b_{6} f\right)$, and the other a G-protein coupled receptor, the human delta opioid receptor (hDOR) $[18,19]$. In this work, we have used PWR spectroscopy to investigate the incorporation of cyt $\mathrm{b}_{6} \mathrm{f}$ and hDOR into a lipid bilayer, and subsequent complex formation with plastocyanin in the former case, and the binding of agonist and antagonist ligands in the latter case. These results provide a good illustration of the ability of PWR spectroscopy to provide useful information about structural events occurring during membrane protein function.

\subsection{Cytochrome $b_{6} f /$ plastocyanin}

The cytochrome $\mathrm{b}_{6} \mathrm{f}$ complex is one of three integral membrane protein complexes involved in electron transport in oxygenic photosynthesis. The complex accepts electrons from the oxygen-producing photosystem II reaction center via plastoquinol, donates electrons to the $\mathrm{NADP}^{+}$-reducing photosystem I reaction center via plastocyanin, and contributes to the electrochemical proton gradient that drives ATP synthesis [20]. The $b_{6} f$ complex consists of four major subunits. Three of them, cytochrome $b_{6}$, cytochrome $\mathrm{f}$, and the Rieske iron-sulfur protein, contain redox prosthetic groups. The fourth subunit is involved together with cytochrome $\mathrm{b}_{6}$ and the Rieske protein in forming the plastoquinol binding site [21]. The redox partners of cytochrome $f$ are its donor, the Rieske protein, and its acceptor, the soluble small $(10.5 \mathrm{kDa})$ copper protein plastocyanin.

The incorporation of cytochrome $\mathrm{b}_{6} \mathrm{f}$ into a solid-supported planar egg PC bilayer can be followed by monitoring PWR spectra obtained after addition of a small aliquot of a concentrated solution of protein, dissolved in an octyl glucoside-containing buffer, to the aqueous compartment of a PWR cell. At low concentrations of the protein (up to $4.2 \mu \mathrm{M}$ ), a shift of the resonance position to a larger angle of incidence occurs (see Fig. 6A, curves 1 and 2). This is accompanied by an increase of the depth and a decrease of the width of the resonance curve as compared to that obtained with the lipid bilayer membrane prior to $b_{6} f$ addition. This is similar to the usual pattern of spectral changes that we have observed previously with other membrane proteins, and results from an increase in mass within the bilayer region due to protein insertion. However, at higher $\mathrm{b}_{6} \mathrm{f}$ concentrations $(6.3 \mu \mathrm{M}$ and $8.4 \mu \mathrm{M}$; Fig. 6A, curves 3 and 4) this pattern is replaced by a reverse shift of the resonance position, with even larger changes in the shape of the resonance curve. This indicates that structural changes are occurring in the proteolipid membrane. Similar patterns were observed with $s$-polarized excitation (not shown).

In order to quantify these spectral changes we have used the following model. In the first stage of incorporation, a simple insertion of cytochrome $b_{6} f$ into the lipid bilayer occurs. This produces a proteolipid structure consisting of two regions in which the hydrophobic components of the $\mathrm{b}_{6} \mathrm{f}$ complex are located within the bilayer interior forming a protein-lipid layer, and the hydrophilic portions of the protein extend outside the lipid membrane and form extramembrane protein-buffer layers. Using this multilayer model, one can fit the experimental resonance curves with theoretical curves (for details see $[5,10]$ ) and 

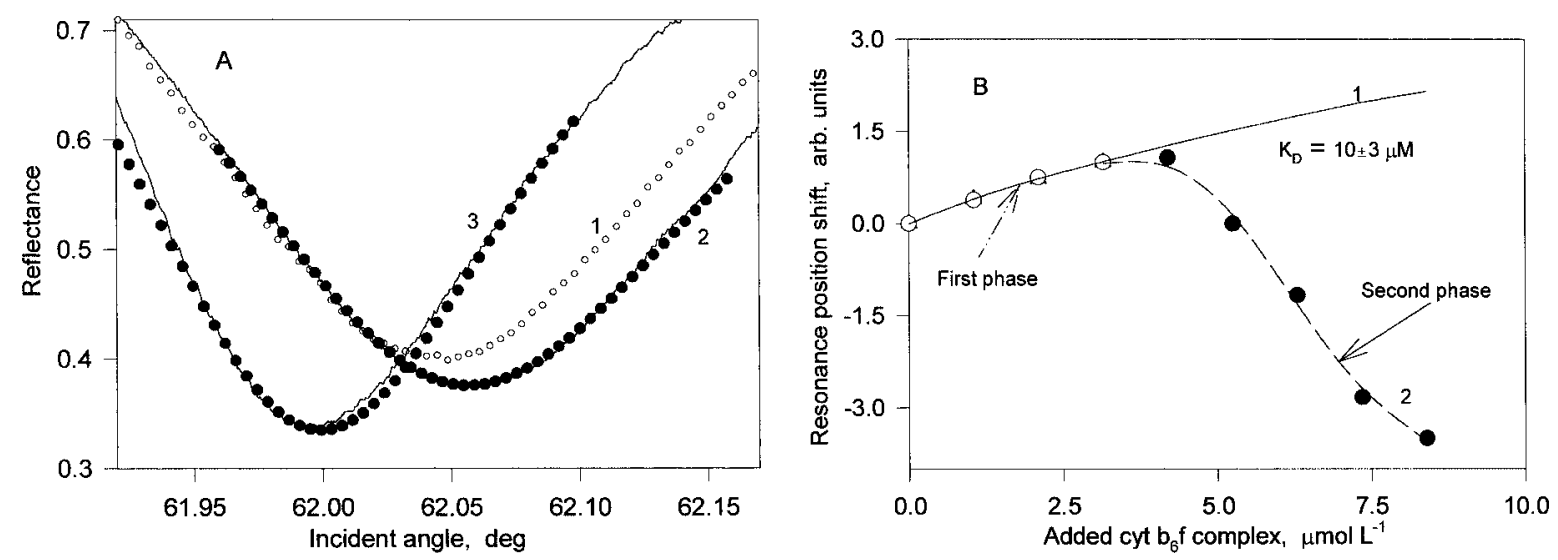

Fig. 6. (A) CPWR spectra obtained with a lipid membrane formed from a solution of $10 \mathrm{mg} / \mathrm{ml}$ egg phosphatidylcholine in butanol containing $1.5 \%$ squalene using $p$-polarized excitation before (open circles in curve 1 ) and after addition of cytochrome $\mathrm{b}_{6} \mathrm{f}$ in octyl glucoside-containing buffer to the aqueous phase (final protein concentration $4.2 \mu \mathrm{M}$, solid line in curve 2; final protein concentration $8.4 \mu \mathrm{M}$; solid line in curve 3). Solid circles correspond to theoretical fits to the experimental spectra. (B) Dependence of the resonance minimum position on the cytochrome $b_{6} f$ complex concentration added to the sample cell. Open circles correspond to the first phase of cytochrome incorporation; solid circles correspond to the second phase of incorporation. Curves show nonlinear least squares fits to either hyperbolic (curve 1) or sigmoidal (curve 2) functions.

thereby obtain the optical parameters of the proteolipid membrane during both phases of protein incorporation. Examples of such theoretical fits are given in Fig. 6A. As is evident, the agreement is quite good.

A plot of the refractive index values obtained from fits to the PWR spectra obtained during the first phase of cytochrome $\mathrm{b}_{6} \mathrm{f}$ incorporation yields a simple hyperbolic curve (apparent $K_{\mathrm{D}}=11 \mu \mathrm{M}$ ). We can also obtain a value for the thickness of the cytochrome $\mathrm{b}_{6} f$ complex from these data $(t=11 \mathrm{~nm}$, corresponding to the sum of values for both protein-lipid and protein-buffer layers). This is in reasonable agreement with existing structural information on the $b_{6} f$ complex. These data support the interpretation that the first phase of protein binding involves simple insertion into the bilayer.

Despite the mass increase due to additional cytochrome $b_{6} \mathrm{f}$ complex incorporation, the total mass of the proteolipid membrane decreases during the second phase of binding. Such a decrease can only be accomplished by transfer of lipid from the bilayer into the plateau-Gibbs border. Furthermore, based on $A_{\mathrm{n}}$ values, during the second phase of protein incorporation the molecular order of the lipid-protein layer drastically decreases. We interpret the spectral changes occurring during this phase to be a result of the dimerization of the $\mathrm{b}_{6} \mathrm{f}$ in the membrane, due to the enhancement by the two-dimensional space of the membrane of the probability of protein-protein interactions. This is consistent with other data showing that cytochrome $b_{6} f$ is capable of forming dimers in solution, and with the plastocyanin binding process as described below.

We have also investigated the interaction between lipid membranes containing the $\mathrm{b}_{6} \mathrm{f}$ complex and plastocyanin added to the aqueous compartment of the PWR cell at the end of both the first and the second phases of cytochrome incorporation. Addition of plastocyanin to the sample at the end of the first $b_{6} f$ incorporation phase produces two different kinds of resonance changes, depending on the plastocyanin concentration. At low plastocyanin concentrations (up to about $25 \mu \mathrm{M}$ ) the resonance position shifts in a similar manner to that which occurs during the second phase of $b_{6} f$ complex incorporation (Fig. 6B), i.e., the resonance minimum shifts to smaller incident angles corresponding to a refractive index decrease. Thus, under these conditions plastocyanin binding to $b_{6} f$ causes the same type of structural changes in 
the membrane as occur in the absence of plastocyanin, although the latter are produced at higher concentrations of the cytochrome. A hyperbolic fit to these data yields a plastocyanin-cytochrome dissociation constant $K_{\mathrm{D}}=10 \mu \mathrm{M}$.

At plastocyanin concentrations higher than $25 \mu \mathrm{M}$ the resonance position (and hence the refractive index) shifts in the opposite direction, i.e., the resonance minimum shifts to higher incident angles as would be expected from a simple binding of plastocyanin to the membrane surface $\left(K_{\mathrm{D}}=170 \mu \mathrm{M}\right)$. This interpretation is confirmed by measuring the binding of plastocyanin to this lipid membrane in the absence of $b_{6} f$ complex. These results demonstrate that plastocyanin can bind to both the $b_{6} f$ complex and to the lipid components of the membrane.

Addition of plastocyanin to the PWR cell at the end of the second phase of $\mathrm{b}_{6} \mathrm{f}$ incorporation results in considerably tighter binding of plastocyanin to the proteolipid membrane $\left(K_{\mathrm{D}}=1 \mu \mathrm{M}\right)$. From a calculation of the masses of cytochrome $\mathrm{b}_{6} \mathrm{f}$ and bound plastocyanin at the end of the second phase of incorporation, we can conclude that two plastocyanin molecules are bound to $a b_{6} f$ dimer. The induction of membrane structural changes by plastocyanin addition at the end of the first phase of $b_{6} f$ incorporation can thus be simply interpreted as reflecting a tighter binding of plastocyanin to the dimeric form of the protein within the membrane, which acts to shift the equilibrium from monomer to dimer.

\subsection{Ligand binding to the human $\delta$-opioid receptor}

The human $\delta$-opioid receptor is a member of a superfamily (containing at least 2,000 members) of seven transmembrane helical G-protein-coupled receptors (GPCR). Opioid receptors mediate pain responses via endogenous ligands such as enkephalins and endorphins and synthetic ligands such as morphine. Generally, activation is achieved by binding an agonist ligand to the receptor on the extracellular side of the membrane, resulting in a change in the ability of a G-protein bound to the receptor on the cytoplasmic side of the membrane to bind and hydrolyze the nucleotide ligand, GTP. Binding an antagonist ligand to the receptor can inhibit such activation. It has been assumed that the ability of ligands to produce these responses is a consequence of conformational changes induced in the receptor molecule by the binding process and transmitted from the external side of the membrane to the cytoplasmic side.

In order to investigate these processes using PWR spectroscopy, we have incorporated the purified receptor into a preformed lipid bilayer, and have monitored the spectral effects of binding of small highly selective ligands to the receptor [19]. These include the agonist DPDPE (a synthetic cyclic pentapeptide) and the antagonist naltrindol (NTI; a synthetic polycyclic peptide analog). We have also evaluated the changes in the receptor structure that accompany these binding interactions. As will be described below, we conclude from these studies that significantly different structural changes are induced in the $\delta$-opioid receptor upon binding these two types of ligands, thereby providing new insights into the structural basis of receptor function.

Receptor molecules were incorporated into a preformed lipid membrane by adding small aliquots of a concentrated solution of the human $\delta$-opioid receptor solubilized in $30 \mathrm{mM}$ octyl glucoside to the aqueous compartment of the PWR cell. Although the overall orientation of the receptor in the bilayer is not known in these experiments, ligand binding to the incorporated receptor occurs efficiently, so that we presume that at least $50 \%$ of the receptors are bound with the liganding site facing the aqueous buffer. Figure 7 shows PWR spectra, obtained with either $p$ - (panel A) or $s$-polarized exciting light (panel B), for a lipid membrane prior to (curve 1) and after addition of an aliquot of detergent-solubilized receptor to the aqueous compartment of the sample cell (curve 2). As with other integral membrane proteins, protein incorporation into the bilayer influences all three parameters of the resonance spectrum, i.e., 

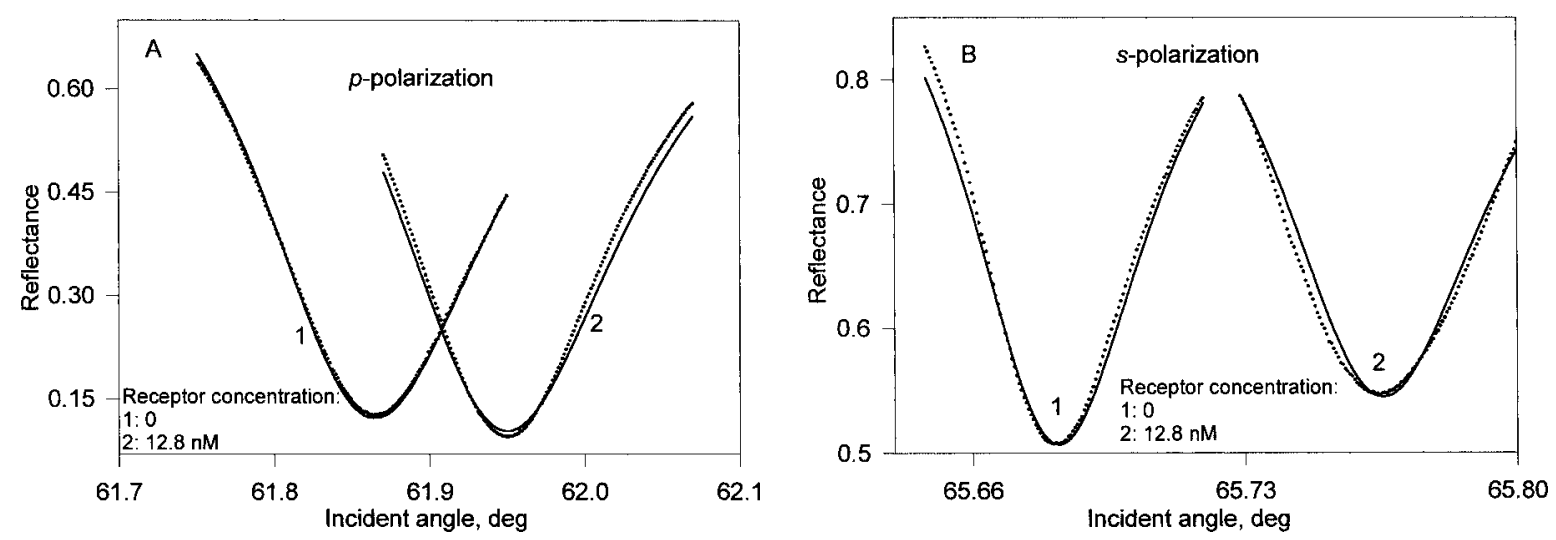

Fig. 7. CPWR spectra obtained with a lipid bilayer containing 75 mol\% egg phosphatidylcholine and 25 mol\% phosphatidylglycerol before (curve 1) and after the addition of human $\delta$-opioid receptor in octyl glucoside-containing buffer (curve 2; final receptor concentration $12.8 \mathrm{nM}$ ) to the aqueous phase. (A) $p$-polarized excitation; (B) $s$-polarized excitation. Dotted curves correspond to theoretical fits to experimental spectra (solid curves).

angular position, depth and spectral half-width. Such changes are due both to mass density changes and to structural alterations of the proteolipid membrane (exemplified by changes in refractive index and thickness).

Figure 8 shows plots of $n, A_{\mathrm{n}}$ and $t$ values derived from fitting data such as these as a function of the concentration of added receptor. These indicate the following:

(1) The process of receptor incorporation is satisfactorily fit by a simple hyperbolic function, yielding an apparent insertion constant of $\approx 14 \mathrm{nM}$. This argues for a quite high efficiency of transfer of receptor from detergent micelle to lipid bilayer.

(2) The extrapolated thickness value $(6.8 \mathrm{~nm})$ describes the dimension of the incorporated protein molecule perpendicular to the membrane plane. This is consistent with the known structural features of the receptor. Extrapolation of the refractive index curves to infinite receptor concentration (Fig. 8A) results in values ( $n_{\mathrm{p} \infty}$ and $n_{\mathrm{s} \infty}$ ) that characterize a monolayer of densely packed receptor molecules. From these one can calculate the surface area occupied by one receptor molecule to be $\approx 1200 \AA^{2}$. This value is also in very good agreement with structural data in the literature.

(3) The increase in the proteolipid membrane anisotropy occurring during the process of receptor incorporation (shown in Fig. 8B), reflects a corresponding increase of the average long-range molecular order in the membrane resulting from receptor-lipid interactions.

When aliquots of ligand solutions are added to the sample cell after receptor incorporation, significant changes in the position, width and depth of the PWR resonance curves occur. These spectral changes reflect the binding of these molecules to the incorporated receptor molecules (control experiments show that no spectral changes are obtained in the absence of receptor). In Fig. 9, examples of resonance spectral shifts obtained with both $p$ - and $s$-polarized exciting light are shown. The data are obtained from experiments in which the agonist is added to the receptor-containing PWR cell first, followed by addition of antagonist, as well as from experiments in which the reverse order of addition was used. As can clearly be seen, the effects of these two types of ligands on the resonance spectral positions are quite different. Thus, the amplitude and direction of the resonance shifts depend upon the type of ligand being bound and the order of addition. It is evident that PWR spectroscopy is able to clearly distinguish between these classes based on these spectral effects. As will be described below, this is a consequence of 

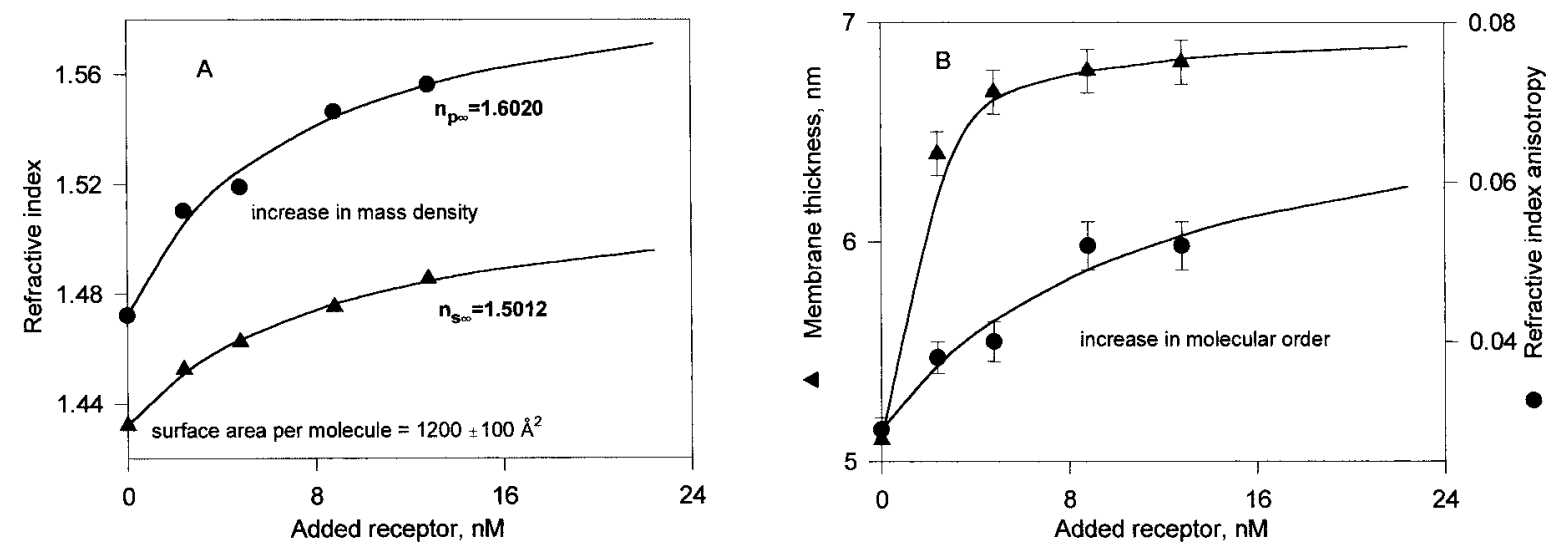

Fig. 8. (A) Refractive index values (circles $p$-polarization; triangles $s$-polarization) for the proteolipid film as a function of added human $\delta$-opioid receptor concentration, obtained from theoretical fits as shown in Fig. 7. Solid curves are hyperbolic fits to the data points; refractive index values extrapolated to infinite receptor concentration are shown in the figure. Refractive index increases reflect increases in mass density. (B) Refractive index anisotropy (circles) and membrane thickness (triangles) values for the proteolipid film as a function of added human $\delta$-opioid receptor concentration, obtained from theoretical fits as shown in Fig. 7. Solid curves are hyperbolic fits to the data points; thickness extrapolated to infinite receptor concentration is shown in the figure. Refractive index anisotropy increase reflects an increase in molecular order upon receptor incorporation.

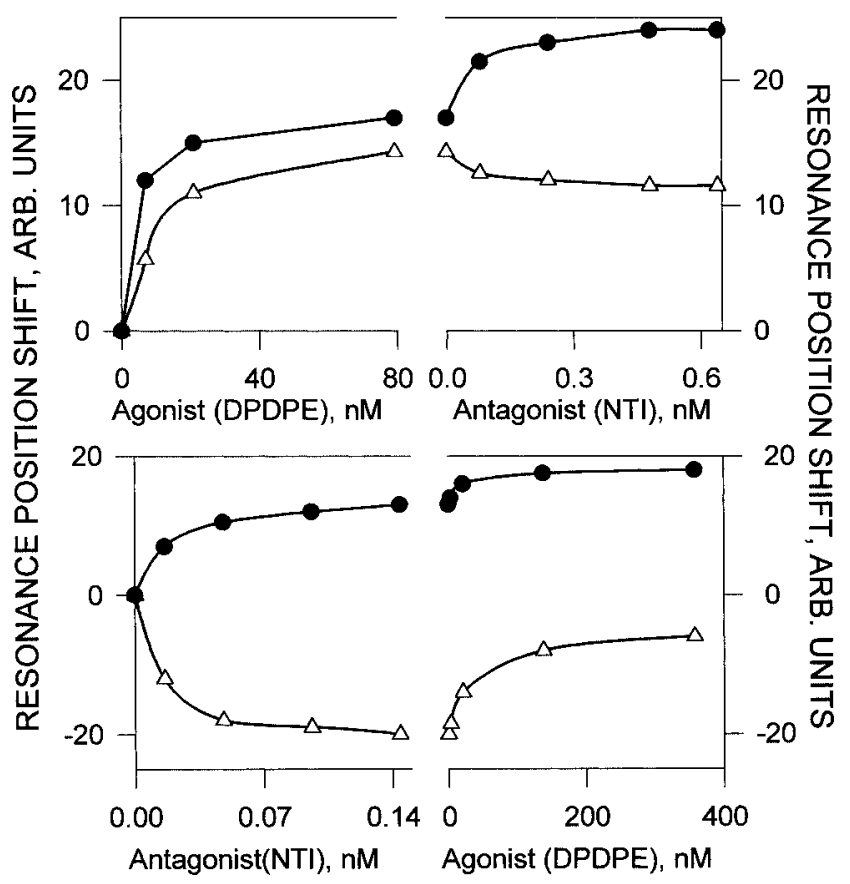

Fig. 9. Dependence of the relative positions of the CPWR spectral minima on the concentrations of added ligand, obtained using either $p$-polarization (closed circles) or $s$-polarization (open triangles). Positive shifts correspond to larger angles of incidence. In these experiments, after $\delta$-opioid receptor incorporation into the lipid membrane, aliquots of the first ligand were added to the sample cell, followed by aliquots of the second ligand. 
the fact that binding of agonists and antagonists produces different conformational states of the receptor. Furthermore, the results clearly demonstrate that an antagonist can shift the receptor from one conformational state to another by displacing an agonist. However, it is also true that adding antagonist after agonist has been bound does not simply reverse the spectral changes generated by agonist binding. In contrast, agonist addition after antagonist is bound is able to reverse the changes caused by the receptorantagonist interaction. As will be described below, this is a direct consequence of the fact that these two types of ligands produce different structural changes in the receptor. It is also important to point out that these spectral changes saturate within concentration ranges that are consistent with literature data for the binding constants of the ligands obtained using radioactively labeled ligands. Furthermore, although the direction of the shifts remains the same regardless of which ligand is added first, the concentration ranges in which the resonance shifts occur depend on the sequence of addition. This is also a consequence of the fact that the ligands produce different conformational states of the receptor. Thus, once a ligand has been bound, a second ligand interacts with a new conformational state of the receptor and therefore is bound less tightly than if it were interacting with an unliganded receptor.

In order to interpret these data in structural terms, it is necessary to extract the optical parameters by theoretically fitting the resonance curves. Figure 10 shows the results obtained from such a procedure as a function of the concentration of added ligand. The solid lines are single hyperbolic curves fitted to the data points. The binding constants obtained from these plots are included in the figure; these are in excellent agreement with values obtained by radioactive ligand binding methods. The following conclusions can be derived from these data:

(1) Agonist binding to the opioid receptor results in an increase in the refractive index anisotropy. This can be ascribed to ordering of the transmembrane helices of the receptor molecule and of the lipid acyl chains in the membrane interior. The increase in membrane thickness indicates that an elongation of the receptor molecule also results from agonist binding. We attribute the mass density increase accompanying binding to an influx of lipid molecules from the plateau-Gibbs border due to membrane curvature caused by this elongation process (note that the ligand mass is too small to account for this increase).

(2) Antagonist binding does not lead to either receptor elongation or mass increase due to lipid influx, but does cause an even larger increase in refractive index anisotropy associated with ordering of transmembrane helices. We conclude from this that binding of this type of ligand produces structural changes that are limited to the transmembrane region of the receptor molecule. This allows a facile explanation of the inability of antagonists to activate the G-protein on the opposite side of the membrane.

\section{Conclusions}

The experiments described above demonstrate the ability of PWR spectroscopy to provide useful insights into the incorporation and functioning of integral membrane proteins. It is important to note that, although in the examples given here the molecular species did not include chromophores absorbing at the plasmon excitation wavelength, and thus the $k$ parameter did not provide useful information, this need not be the case. A molecular labeling strategy to introduce such chromophores can greatly expand the utility of the method (for details see [9,12]). It is also important to point out that, although the structural information provided by this technique is at a low level of resolution, this is compensated by the ability of PWR to observe a single lipid bilayer in real time under physiological conditions of concentration 


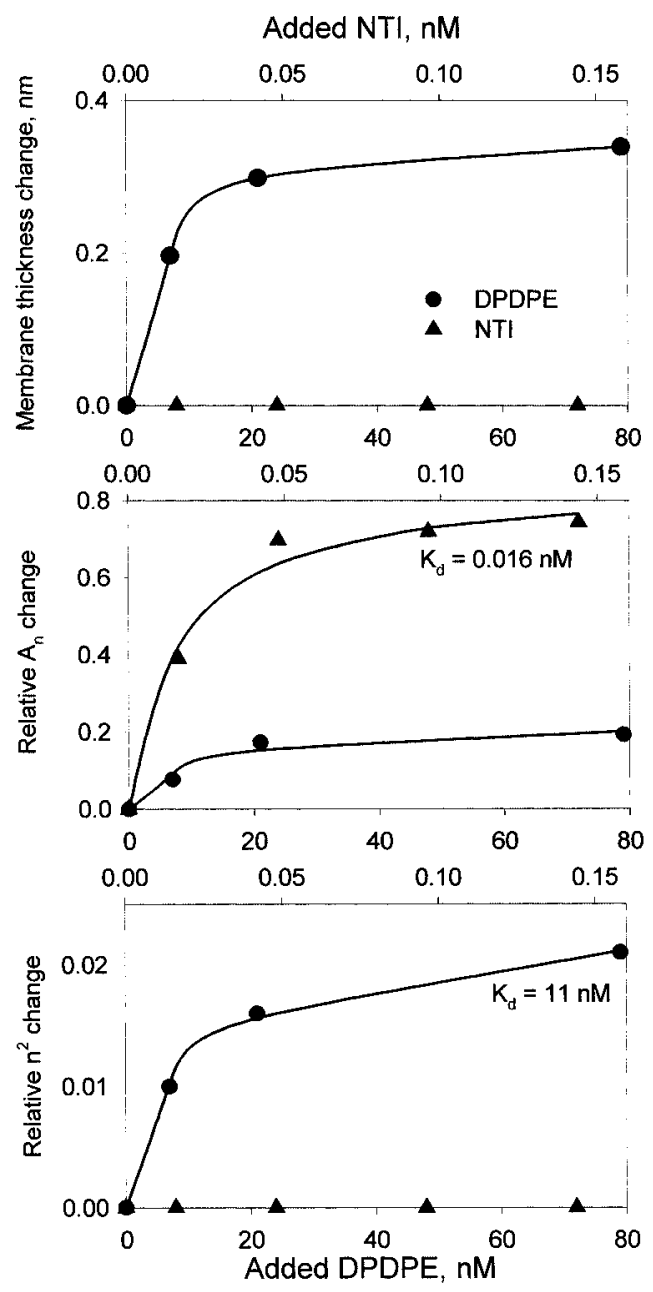

Fig. 10. Changes in optical parameters induced by ligand binding to the human $\delta$-opioid receptor. (A) membrane thickness changes; (B) relative changes in refractive index (these are expressed as $n^{2}$, which is proportional to changes in mass density); (C) relative changes in refractive index anisotropy. Values obtained by theoretical fits to CPWR spectra, as shown in Fig. 7. Solid curves are hyperbolic fits to the data points; in some cases $K_{\mathrm{d}}$ values are shown in the figure.

and environment. As has been demonstrated above, the structural information obtained with PWR is required in order to obtain appropriate insights into molecular interactions occurring during the functional activity of membrane-associated proteins. In contrast, conventional SPR is unable to provide such structural information and therefore its application can only be useful with isotropic systems that cannot mimic the processes occurring in biomembranes. It should also be kept in mind that PWR studies are by no means limited to biomembrane systems. Thus, any method of molecular immobilization onto a plasmon resonator surface will suffice. In this context, the dielectric coating of the plasmon-generating metal film provides a convenient medium for attaching a wide variety of molecules to the PWR device, thereby greatly expanding the range of systems that can be investigated. Furthermore, the evanescent field generated during plasmon excitation can be used to excite fluorescence from appropriate molecular species, thereby increasing the detection protocols that can be used. All of these characteristics should allow PWR spectroscopy to be of value to a number of different areas of science. 


\section{Acknowledgements}

This work was supported in part by grants from the National Science Foundation (MCB-9904753) and the National Institutes of Health (1 R01 GM59630).

\section{References}

[1] S. Löfás and S.B. Johnsson, A novel hydrogel matrix on gold surfaces in surface plasmon resonance sensors for fast and efficient covalent immobilization of ligands, J. Chem. Soc. Chem. Commun. (1991), 1526-1528.

[2] E. Stenberg, B. Persson, H. Roos and C. Urbaniczky, Quantitative determination of surface concentration of protein with surface plasmon resonance using radiolabeled proteins, J. Colloid Interface Sci. 143 (1991), 513-520.

[3] M. Hendrix, E.S. Priestley, G.F. Joyce and C.-H. Wong, Direct observation of aminoglycosyl-RNA interactions by surface plasmon resonance, J. Am. Chem. Soc. 119 (1997), 3641-3648.

[4] Z. Salamon and G. Tollin, Surface plasmon resonance spectroscopy: a new biophysical tool for probing membrane structure and function, in: Biomembrane Structures, P.I. Haris and D. Chapman, eds, IOS Press, Amsterdam, 1998, pp. 186204.

[5] Z. Salamon, H.A. Macleod and G. Tollin, Surface plasmon resonance spectroscopy as a tool for investigating the biochemical and biophysical properties of membrane protein systems. I. Theoretical principles, Biochim. Biophys. Acta 1331 (1997), 117-129.

[6] Z. Salamon, H.A. Macleod and G. Tollin, Surface plasmon resonance spectroscopy as a tool for investigating the biochemical and biophysical properties of membrane protein systems. II. Applications to biological systems, Biochim. Biophys. Acta 1331 (1997), 131-152.

[7] S. Heyse, T. Stora, E. Schmid, J.H. Lakey and H. Vogel, Emerging techniques for investigating molecular interactions at lipid membranes, Biochim. Biophys. Acta 1376 (1998), 319-338.

[8] Z. Salamon, H.A. Macleod and G. Tollin, Coupled plasmon-waveguide resonators: a new spectroscopic tool for probing proteolipid film structure and properties, Biophys. J. 73 (1997), 2791-2797.

[9] Z. Salamon, M.F. Brown and G. Tollin, Plasmon resonance spectroscopy: probing interactions within membranes, Trends Biochem. Sci. 24 (1999), 213-219.

[10] Z. Salamon and G. Tollin, Surface plasmon resonance: theoretical principles, in: Encyclopedia of Spectroscopy and Spectrometry, Vol. 3, J.C. Lindon, G.E. Tranter and J.L. Holmes, eds, Academic Press, New York, 1999, pp. 2311-2319.

[11] Z. Salamon and G. Tollin, Surface plasmon resonance: applications, in: Encyclopedia of Spectroscopy and Spectrometry, Vol. 3, J.C. Lindon, G.E. Tranter and J.L. Holmes, eds, Academic Press, New York, 1999, pp. 2294-2302.

[12] Z. Salamon and G. Tollin, Optical anisotropy in lipid bilayer membranes: coupled plasmon-waveguide resonance measurements of molecular orientation, polarizability, and shape, Biophys. J. 80 (2001), 1557-1567.

[13] Z. Salamon and G. Tollin, Surface plasmon resonance spectroscopy in peptide and protein analysis, in: Encyclopedia of Analytical Chemistry, R.A. Meyers, ed., John Wiley \& Sons Ltd., Chichester, 2000, pp. 6050-6061.

[14] P. Mueller, D.O. Rudin, H.T. Tien and W.C. Wescott, Reconstitution of cell membrane structure in vitro and its transformation into excitable system, Nature 194 (1962), 979-980.

[15] Z. Salamon, Y. Wang, M.F. Brown, H.A. Macleod and G. Tollin, Conformational changes in rhodopsin probed by surface plasmon resonance spectroscopy, Biochemistry 33 (1994), 13 706-13 711.

[16] Z. Salamon, Y. Wang, J.L. Soulages, M.F. Brown and G. Tollin, Surface plasmon resonance spectroscopy studies of membrane proteins: transducin binding and activation by rhodopsin monitored in thin membrane films, Biophys. J. 71 (1996), 283-294.

[17] Z. Salamon and G. Tollin, Surface plasmon resonance studies of complex formation between cytochrome c and bovine cytochrome c oxidase incorporated into a supported planar lipid bilayer: II. Binding of cytochrome c to oxidase-containing cardiolipin/phosphatidylcholine membranes, Biophys. J. 71 (1996), 858-867.

[18] Z. Salamon, D. Huang, W.A. Cramer and G. Tollin, Coupled plasmon-waveguide resonance spectroscopy studies of the cytochrome b $b_{6}$ f/plastocyanin system in supported lipid bilayer membranes, Biophys. J. 75 (1998), 1874-1885.

[19] Z. Salamon, S. Cowell, E. Varga, H.I. Yamamura, V.J. Hruby and G. Tollin, Plasmon resonance studies of agonist/antagonist binding to the human $\delta$-opioid receptor: new structural insights into receptor-ligand interactions, Biophys. J. 79 (2000), 2463-2474.

[20] W.A. Cramer, S.E. Martinez, P.N. Furbacher, D. Huang and J.L. Smith, The cytochrome b ${ }_{6}$ f complex, Curr. Opin. Struct. Biol. 4 (1994), 536-544.

[21] W.A. Cramer, G.M. Soriano, M. Ponomarev, D. Huang, H. Zhang, S.E. Martinez and J.L. Smith, Some new structural aspects and old controversies concerning the cytochrome $\mathrm{b}_{6} \mathrm{f}$ complex of oxygenic photosynthesis, Annu. Rev. Plant Physiol. Plant Mol. Biol. 47 (1996), 477-508. 


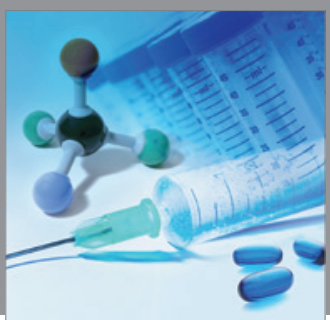

International Journal of

Medicinal Chemistry

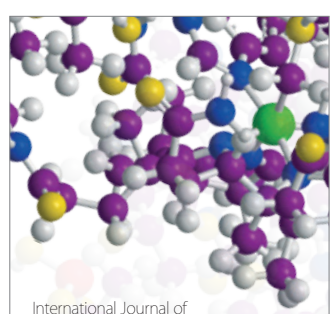

Carbohydrate Chemistry

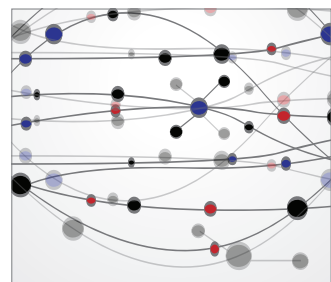

The Scientific World Journal
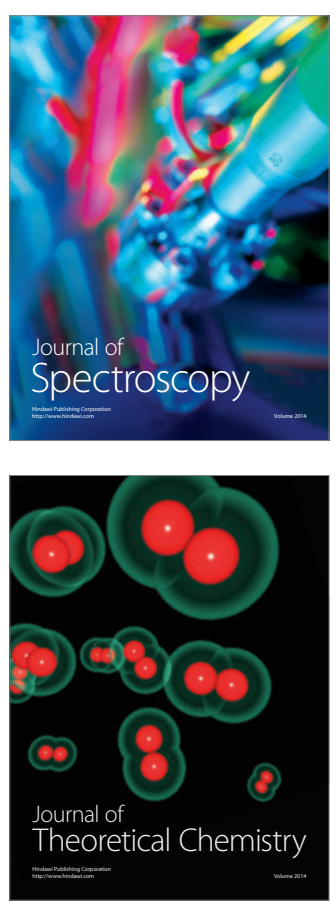
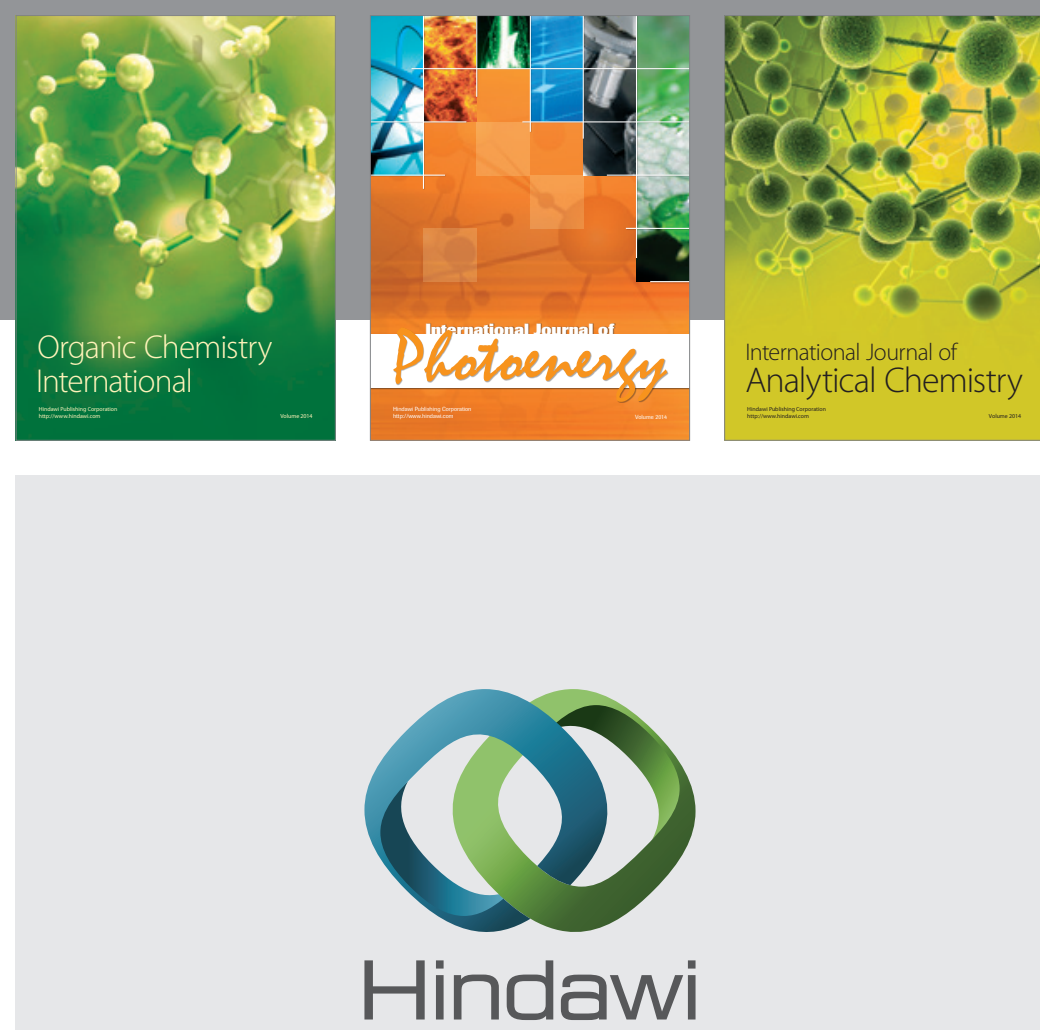

Submit your manuscripts at

http://www.hindawi.com
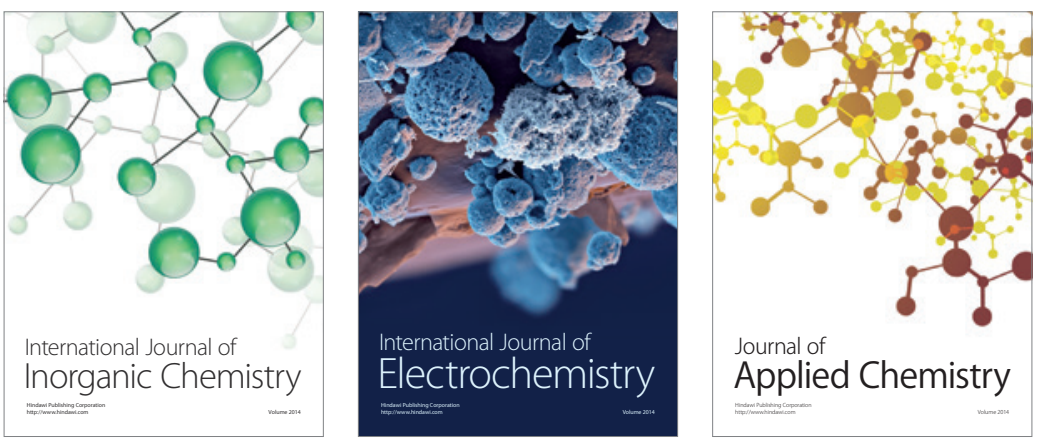

Journal of

Applied Chemistry
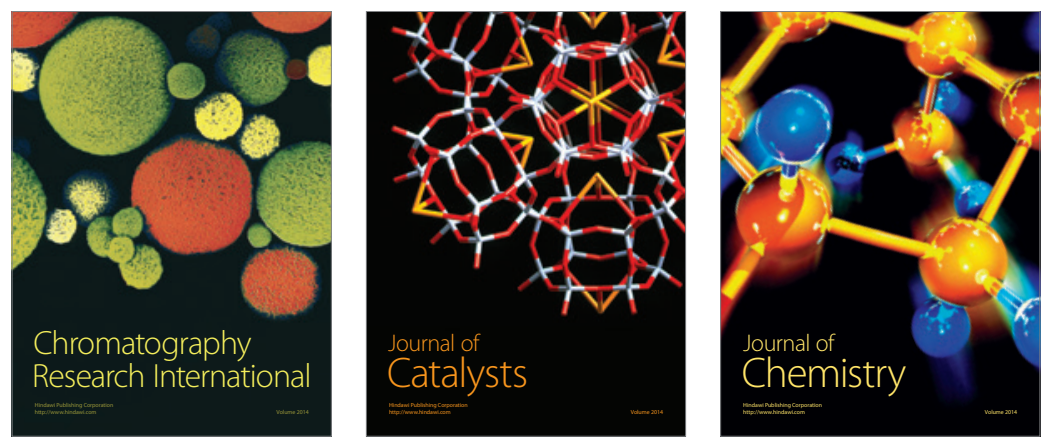
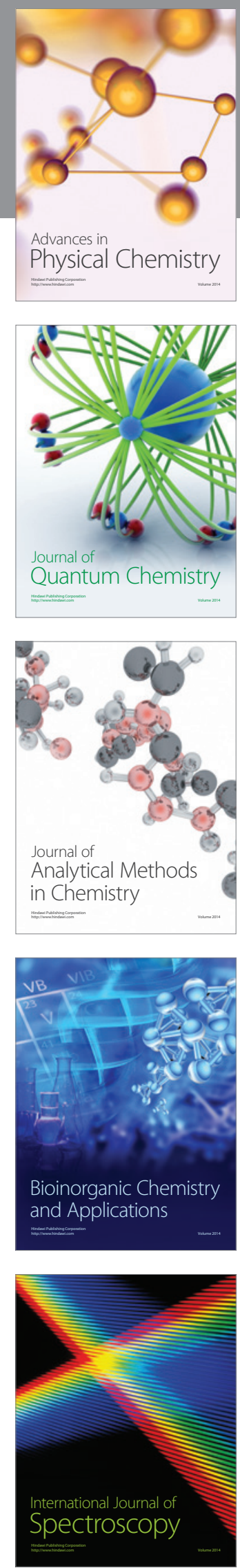